\title{
New Quantum Error-Correcting Codes from Hermitian Self-Orthogonal Codes over GF(4)
}

Jon-Lark Kim

Department of Mathematics, Statistics, and Computer Science, $322 \mathrm{SEO}(\mathrm{M} / \mathrm{C} 249)$,

University of Illinois-Chicago,

851 S. Morgan, Chicago, IL 60607-7045, USA

\begin{abstract}
In order to construct good quantum-error-correcting codes, we construct good Hermitian self-orthogonal linear codes over GF(4). In this paper we construct record-breaking pure quantum-error-correcting codes of length 24 with 2 encoded qubits and minimum weight 7 from Hermitian self-orthogonal codes of length 24 with dimension 11 over GF(4). This shows that length $n=24$ is the smallest length for any known $[[n, k, d]]$ quantum-error-correcting code with $k \geq 2$ and $d=7$. We also give a construction method to produce Hermitian self-orthogonal linear codes GF(4) from a shorter length such code.
\end{abstract}

\section{Introduction}

It was shown [4] in 1995 that there could exist quantum-error-correcting codes (QECC throughout the paper) which would protect quantum information as classical error-correcting codes protect classical information. See [1] for the brief history of QECC. It is also known [1] that the problem of finding QECC is transformed into the problem of finding additive self-orthogonal codes under a certain inner product over the finite field GF(4). These additive self-orthogonal codes include the classical Hermitian self-orthogonal codes over GF(4). So our purpose is to construct good Hermitian self-orthogonal codes in order to construct good QECC using the ideas of [3].

We recall some basic definitions from $[1,2]$. Let $\operatorname{GF}(4)=\{0,1, \omega, \bar{\omega}\}$ with the convention that $2=\omega$ and $3=\bar{\omega}$ where $\bar{\omega}=\omega^{2}=1+\omega$. An additive code $\mathcal{C}$ over $\mathrm{GF}(4)$ of length $n$ is an additive subgroup of $\mathrm{GF}(4)^{\mathrm{n}}$. As $\mathcal{C}$ is a free $\mathrm{GF}(2)$-module, it has size $2^{k}$ for some $0 \leq k \leq 2 n$. We call $\mathcal{C}$ an $\left(n, 2^{k}\right)$ code. It has a basis, as a $\mathrm{GF}(2)$-module, consisting of $k$ basis vectors; a generator matrix of $\mathcal{C}$ will be a $k \times n$ matrix with entries in $\mathrm{GF}(4)$ whose rows are a basis of $\mathcal{C}$. The weight $\mathrm{wt}(\mathbf{c})$ of $\mathbf{c} \in \mathcal{C}$ is the number of nonzero components of $\mathbf{c}$. The minimum weight $d$ of $\mathcal{C}$ is the smallest weight of any nonzero codeword in $\mathcal{C}$. If $\mathcal{C}$ is an $\left(n, 2^{k}\right)$ additive code of minimum weight $d, \mathcal{C}$ is called an $\left(n, 2^{k}, d\right)$ code.

To study QECC, we consider a somewhat different inner product from the ordinary inner product. We start with the following trace map. The trace 
map $\operatorname{Tr}: \mathrm{GF}(4) \rightarrow \mathrm{GF}(2)$ is given by

$$
\operatorname{Tr}(x)=x+x^{2} .
$$

In particular $\operatorname{Tr}(0)=\operatorname{Tr}(1)=0$ and $\operatorname{Tr}(\omega)=\operatorname{Tr}(\bar{\omega})=1$. The conjugate of $x \in \mathrm{GF}(4)$, denoted $\bar{x}$, is the image of $x$ under the Frobenius automorphism; in other words, $\overline{0}=0 \overline{1}=1$, and $\overline{\bar{\omega}}=\omega$. We now define the trace inner product of two vectors $\mathbf{x}=x_{1} x_{2} \cdots x_{n}$ and $\mathbf{y}=y_{1} y_{2} \cdots y_{n}$ in $\operatorname{GF}(4)^{\mathrm{n}}$ to be

$$
\mathbf{x} \star \mathbf{y}=\sum_{i=1}^{n} \operatorname{Tr}\left(x_{i} \overline{y_{i}}\right) \in \mathrm{GF}(4) .
$$

If $\mathcal{C}$ is an additive code, its dual, denoted $\mathcal{C}^{\perp}$, is the additive code $\{\mathbf{x} \in$ $\operatorname{GF}(4)^{\mathrm{n}} \mid \mathbf{x} \star \mathbf{c}=0$ for all $\left.\mathbf{c} \in \mathcal{C}\right\}$. If $\mathcal{C}$ is an $\left(n, 2^{k}\right)$ code, then $\mathcal{C}^{\perp}$ is an $\left(n, 2^{2 n-k}\right)$ code. As usual, $\mathcal{C}$ is trace self-orthogonal if $\mathcal{C} \subseteq \mathcal{C}^{\perp}$ and self-dual if $\mathcal{C}=\mathcal{C}^{\perp}$. In particular, if $\mathcal{C}$ is trace self-dual, $\mathcal{C}$ is an $\left(n, 2^{n}\right)$ code.

We say that two additive codes $\mathcal{C}_{1}$ and $\mathcal{C}_{2}$ are equivalent provided there is a map sending the codewords of $\mathcal{C}_{1}$ onto the codewords of $\mathcal{C}_{2}$ where the map consists of a permutation of coordinates followed by a scaling of coordinates by elements of $G F(4)$ followed by conjugation of some of the coordinates. Notice that permuting coordinates, scaling coordinates, and conjugating some coordinates of a self-orthogonal (or self-dual) code does not change self-orthogonality (or self-duality) and the weight distribution of the code. The automorphism group of $\mathcal{C}$, denoted $\operatorname{Aut}(\mathcal{C})$, consists of all maps which permute coordinates, scale coordinates, and conjugate coordinates that send codewords of $\mathcal{C}$ to codewords of $\mathcal{C}$.

Now we state the relationship between QECC and additive self-orthogonal codes over GF(4).

Lemma 1 (Theorem 2, [1]). Suppose that $\mathcal{C}$ is an additive trace selforthogonal $\left(n, 2^{n-k}\right)$ code of $\mathrm{GF}(4)^{\mathrm{n}}$ such that there are no vectors of weight $<d$ in $\mathcal{C}^{\perp} \backslash \mathcal{C}$. Then an additive quantum-error-correcting code with parameters $[[n, k, d]]$ is obtained.

If there are no nonzero vectors of weight $<d$ in $\mathcal{C}^{\perp}$ in the above lemma, $\mathcal{C}$ is pure (or nondegenerate); otherewise it is impure (or degenerate) [1]. A $[[n, k, d]]$ QECC can correct $[(d-1) / 2]$ errors, where $k$ is the number of encoded qubits (quantum bits).

The Hermitian inner product is defined as

$$
\mathbf{x} \cdot \mathbf{y}=x_{1} \overline{y_{1}}+\cdots+x_{n} \overline{y_{n}} \in \mathrm{GF}(4),
$$

for two vectors $\mathbf{x}=\left(x_{1}, \cdots, x_{n}\right)$ and $\mathbf{y}=\left(y_{1}, \cdots, y_{n}\right)$ in $\mathrm{GF}(4)^{\mathrm{n}}$. A linear code with length $n$, dimension $k$ (as a vector space over GF(4)), and minimum weight $d$ is called an $[n, k, d]$ code. The following theorem explains why Hermitian self-orthogonal linear codes are interesting in order to construct QECC. 
Lemma 2 (Theorem 3, [1]). A linear code $\mathcal{C}$ is self-orthogonal with respect to (1) if and only if it is self-orthogonal with respect to (2).

Combining the above two lemmas, we get the following corollary.

Corollary $1([\mathbf{1}, \mathbf{5}])$. Let $\mathcal{C}$ be a Hermitian self-orthogonal linear $[n, k]$ code over $\mathrm{GF}(4)$ such that there are no vectors of weight $<d$ in $\mathcal{C}^{\perp} \backslash \mathcal{C}$, where $\mathcal{C}^{\perp}$ is the Hermitian dual of $\mathcal{C}$. Then there is a quantum-error-correcting $[[n, n-2 k, d]]$ code.

Proof. Since the given code $\mathcal{C}$ is linear, it has parameters as an additive code $\left(n, 2^{2 k}\right)=\left(n, 2^{n-(n-2 k)}\right)$. Thus by Lemma 1 a quantum-error-correcting $[[n, n-2 k, d]]$ code is obtained.

\section{Construction method}

By generalizing the building-up construction [3, Theorem 1] for self-dual codes over GF(4) to self-orthogonal codes, we have the following theorem. We remark that there was an error in [3, Theorem 1] about the definition of $\overline{y_{i}}$ and so correct it here.

Theorem 1. Let $G_{0}=\left(g_{i}\right)$ be a generator matrix(may not be in standard form) of a Hermitian self-orthogonal code $\mathcal{C}_{0}$ over $\mathrm{GF}(4)$ of length $n$ with dimension $k$, where $g_{i}$ are rows of $G_{0}$ respectively for $1 \leq i \leq k$. Let $\mathbf{x}=$ $\left(x_{1}, \cdots, x_{n}\right)$ be a vector in $\mathrm{GF}(4)^{\mathrm{n}}$ with an odd weight. Suppose that $\overline{y_{i}}:=$ $\left(x_{1}, \cdots, x_{n}\right) \cdot g_{i}$ for $1 \leq i \leq k$. Here $\overline{y_{i}}$ is the conjugate of $y_{i}$ and $\cdot$ denotes the Hermitian inner product. Then the following matrix

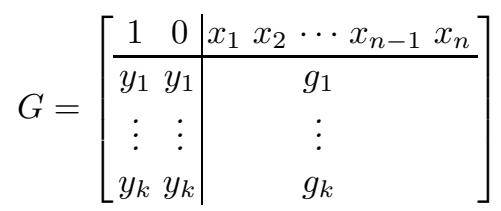

generates a Hermitian self-orthogonal code $\mathcal{C}$ over $\mathrm{GF}(4)$ of length $n+2$ with dimension $k+1$.

As an example of the above theorem, let $\mathcal{C}_{0}$ be a Hermitian self-dual code over $\mathrm{GF}(4)$ generated by $\{1010,0101\}$. If we take $\mathbf{x}=(01 \omega \bar{\omega})$, then the code $\mathcal{C}$ is generated by $\{1001 \omega \bar{\omega}, \overline{\omega \omega} 1010, \overline{\omega \omega} 0101\}$ by Theorem 1 . This is the unique $[6,3,4]$ Hexacode over GF(4).

As in [3, Theorem 2] we get the converse of the above theorem as follows.

Theorem 2. Any Hermitian self-orthogonal code $\mathcal{C}$ over $\mathrm{GF}(4)$ of length $n$ and dimension $k>1$ with minimum weight $d>2$ is obtained from some Hermitian self-orthogonal code $\mathcal{C}_{0}$ of length $n-2$ and dimension $k-1$ (up to equivalence) by the construction in Theorem 1.

In the following section, we construct 19 inequivalent linear Hermitian selforthogonal $[24,11,8]$ codes over GF(4) with its dual minimum weight 7 . These give record-breaking $[[24,2,7]]$ quantum-error-correcting codes. 
Table 1. Generator matrix of $Q_{22}^{1}$

$$
G\left(Q_{22}^{1}\right)=\left[\begin{array}{l}
1000000100000133233203 \\
0100000300020221231212 \\
0010000100033303000120 \\
0001000200012220332002 \\
0000100200021031201103 \\
0000010200021001233210 \\
0000001100022020312101 \\
0000000010000100113322 \\
000000000100000111111 \\
0000000000100010112233
\end{array}\right]
$$

Table 2. New [[24, 2, 7]] quantum-error-correcting codes using $Q_{22}^{1}$

\begin{tabular}{c|c|c||c|c|c}
\hline codes $C$ & $\mathbf{x}=\left(x_{1}, \cdots, x_{11}\right)$ & $A_{8}, B_{7}$ & codes $C$ & $\mathbf{x}=\left(x_{1}, \cdots, x_{11}\right)$ & $A_{8}, B_{7}$ \\
\hline$Q_{24,1}$ & 0300111121 & 117,171 & $Q_{24,2}$ & 22321301221 & 144,156 \\
$Q_{24,3}$ & 22131000321 & 141,186 & $Q_{24,4}$ & 10020033021 & 108,174 \\
$Q_{24,5}$ & 13013111132 & 99,156 & $Q_{24,6}$ & 12030021132 & 120,198 \\
$Q_{24,7}$ & 23310200132 & 105,132 & $Q_{24,8}$ & 20012000332 & 96,150 \\
$Q_{24,9}$ & 31100212032 & 105,165 & $Q_{24,10}$ & 02212022032 & 102,162 \\
$Q_{24,11}$ & 12010313032 & 126,183 & $Q_{24,12}$ & 11110021202 & 114,150 \\
$Q_{24,13}$ & 20223012202 & 96,159 & $Q_{24,14}$ & 33030202002 & 105,147 \\
$Q_{24,15}$ & 02311200002 & 108,147 & $Q_{24,16}$ & 31231302123 & 102,150 \\
$Q_{24,17}$ & 33321333303 & 102,159 & $Q_{24,18}$ & 20212031120 & 108,180 \\
$Q_{24,19}$ & 21121332320 & 90,144 & & & \\
\hline
\end{tabular}

\section{Existence of $[[24,2,7]]$ quantum-error-correcting codes}

According to [1, Table III], it is known that the highest minimum weight $d$ for $[[24,2, d]]$ codes is bounded by $6 \leq d \leq 8$. We apply Theorem 1 to a Hermitian self-orthogonal $[22,10,8]$ code $Q_{22}^{1}$ in Table 1 with many possibilities for vectors $\mathbf{x}$ to get 19 inequivalent Hermitian self-orthogonal $[24,11,8]$ codes such that their dual codes all have minimum weight $d=7$. Hence it follows from Corollary 1 that there exist pure [[24,2,7]] codes. Moreover length $n=24$ is the smallest length for any known three error-correcting $[[n, k, 7]]$ codes with $k \geq 2$ according to [1, Table III]. See Table 2 for such codes, where $A_{8}$ (resp, $B_{7}$ ) denotes the number of minimum vectors in $\mathcal{C}\left(\right.$ resp, $\left.\mathcal{C}^{\perp}\right)$, justifying the inequivalence of the codes. Here we gave the vectors $\mathbf{x}$ with only first 11 co- 
ordinates, the right half being 1's. For example, $\mathbf{x}=(23310200132)$ in $Q_{24,7}$ means $\mathbf{x}=(2331020013211111111111)$. We summarize our result as follows.

Theorem 3. There exist at least 19 inequivalent pure $[[24,2,7]]$ quantumerror-correcting codes, which are obtained from Hermitian self-orthogonal linear $[24,11,8]$ codes with its dual minimum weight $\%$.

Acknowledgment. The author would like to thank Vera Pless for reading the first manuscript and the referee for useful comments.

\section{References}

1. Calderbank, A. R., Rains, E. M., Shor, P. W., Sloane, N. J. A. (1998) Quantum error correction via codes over GF(4). IEEE Trans. Inform. Theory. 44, 13691387

2. Gaborit, P., Huffman, W. C., Kim, J.-L., and Pless, V. (2001) On additive GF(4) codes. DIMACS Workshop on Codes and Association Schemes DIMACS Series in Discrete Math. and Theoret. Computer Science, American Mathematical Society, 56, 135-149

3. Kim, J.-L. (2001) New self-dual codes over GF(4) with the highest known minimum weights. IEEE Trans. Inform. Theory. 47, 1575-1580

4. Shor, P. W. (1995) Scheme for reducing decoherence in quantum memory. Phys. Rev. A. 52, 2493

5. Thangaraj, A., McLaughlin, S. W. (2001) Quantum codes from cyclic codes over GF $\left(4^{\mathrm{m}}\right)$. IEEE Trans. Inform. Theory. 47, 1176-1178 